$\Rightarrow$ NANOTECHNOLOGY

\section{Tag teams}

Nanoparticles are being used to address the long-standing problem of delivering and activating a cytotoxic drug specifically in tumours. Two recent papers have exploited some of the properties of nanoparticles to efficiently kill tumour cells in vivo.

Geoffrey von Maltzahn, Sangeeta N. Bhatia and colleagues have developed a nanotechnological tag team that makes use of the coagulation cascade. The authors first showed that heating, using a $45-53^{\circ} \mathrm{C}$ water bath, an MDA-MB-435 xenograft on the flank of a nude mouse can induce coagulation and fibrin accumulation in the xenograft. They then investigated whether polyethylene glycol (PEG)-coated gold nanorods (PEGNRs), which passively accumulate in tumours and which can convert external electromagnetic energy into heat, could specifically induce tumour coagulation. Mice were intravenously injected with PEGNRs, and 72 hours post-injection, when excess PEG-NRs had cleared the circulation, one of a bilateral set of MDA-MB-435 xenografts was exposed to near-infrared (NIR) light as a source of electromagnetic energy. This induced substantial coagulation only in the treated xenograft. They also assessed a tumour targeted, truncated form of human tissue factor (tTF) that contains an RGD peptide that will bind to angiogenic integrin $\left(\alpha_{\mathrm{v}} \beta_{3}\right)$ receptors. The presence of $\mathrm{tTF}$ triggers the coagulation cascade, and this also resulted in substantial coagulation in tumour vessels. The second half of the tag team - magnetofluorescent iron oxide nanoworms, as a potential imaging agent, and

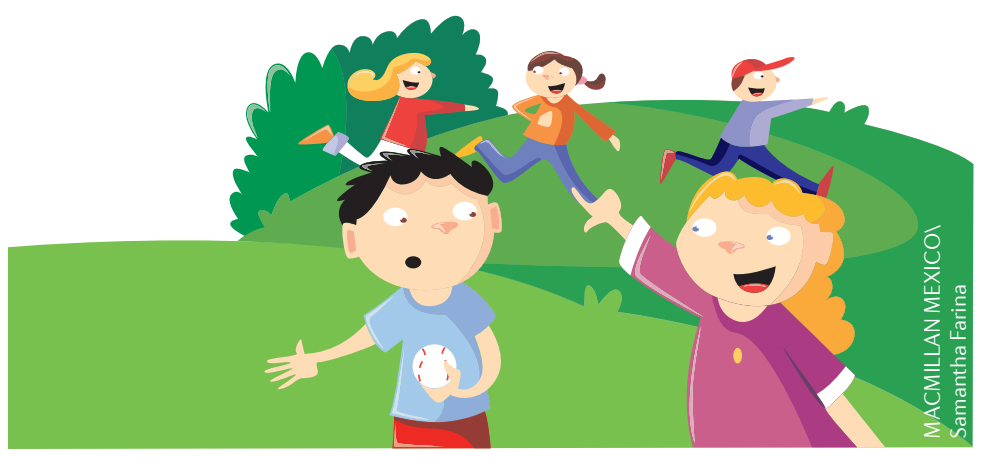

doxorubicin-loaded liposomes, as an example of a cytotoxic agent - can communicate with the first half by virtue of peptide coats that recognize fibrin (produced by coagulation) or that function as a substrate of the coagulation transglutaminase, factor XIII (FXIII). Thus, when coagulation in the tumour is induced by the first nanoparticle, the second nanoparticle is recruited in large quantities specifically to the tumour.

To fully test their system, the authors initially combined PEG-NRs with FXIII-coupled nanoworms. Exposure of the xenografts to NIR light to activate the PEG-NRs induced the substantial accumulation of the nanoworms compared with xenografts that were not exposed to NIR light, as well as surrounding tissues. So, can this technology be used to deliver cytotoxic agents specifically to tumours? The combination of PEG-NRs with FXIII-coupled doxorubicin liposomes resulted in doxorubicin concentrations of greater than 40-fold in xenografts compared with doxorubicin liposomes alone. A single treatment of PEG-NR with FXIII-coupled doxorubicin liposomes showed prolonged inhibition of tumour growth.

One current drawback is that the conversion of electromagnetic energy into heat by conventional metal oxide nanoparticles is inefficient, so larger amounts of nanoparticles are needed, and this could lead to side effects. Jae-Hyun Lee and colleagues looked at the physical properties of various nanoparticles and came up with a design consisting of a magnetically hard core surrounded by a magnetically soft shell. This design allows for a more efficient conversion of electromagnetic waves into heat. Injection of nanoparticles consisting of a core of $\mathrm{CoFe}_{2} \mathrm{O}_{4}$, and a shell of $\mathrm{MnFe}_{2} \mathrm{O}_{4}\left(\mathrm{CoFe}_{2} \mathrm{O}_{4} @ \mathrm{MnFe}_{2} \mathrm{O}_{4}\right)$ into U87MG xenografts followed by a 10 minute exposure to an alternating current magnetic field, resulted in tumour ablation after 1 month and compared favourably to mice treated with $75 \mu \mathrm{g}$ of doxorubicin, in which the xenografts regrew. Complete tumour ablation required $75 \mu \mathrm{g}$ of $\mathrm{CoFe}_{2} \mathrm{O}_{4} @ \mathrm{MnFe}_{2} \mathrm{O}_{4}$ nanoparticles compared with a high dose $(300 \mu \mathrm{g})$ of doxorubicin. The mice exhibited no side effects from exposure to the magnetic field.

Both of these papers illustrate how modifications of nanoparticles should hopefully translate into more effective tumour treatments.

\section{Nicola McCarthy}

ORIGINAL RESEARCH PAPERS von Maltzahn, G et al. Nanoparticles that communicate in vivo to amplify tumour targeting. Nature Mater. 10 , 545-552 (2011) | Lee, J.-H. et al. Exchange-coupled magnetic nanoparticles for efficient heat induciton. Nature Nano. 26 Jun 2011 (doi: 10.1038/ nnano.2011.95) 